\title{
Vibrational Circular Dichroism Shows Unusual Sensitivity to Protein Fibril Formation and Development in Solution
}

\author{
Shengli Ma, ${ }^{\mathrm{a}}$ Xiaolin $\mathrm{Cao}^{\mathrm{a}, 1}$, Mimi Mak, ${ }^{\mathrm{a}}$ Adeola Sadik, ${ }^{\mathrm{a}}$ Christoph Walkner, ${ }^{\mathrm{a}}$ Teresa B. Freedman, ${ }^{\mathrm{a}}$ \\ Igor K. Lednev, ${ }^{b}$ Rina K. Dukor ${ }^{c}$ and Laurence A. Nafie ${ }^{a, c_{*}}$ \\ ${ }^{a}$ Department of Chemistry, Syracuse University, Syracuse, New York, 13244 \\ ${ }^{b}$ Department of Chemistry, University at Albany, SUNY, Albany, New York, 12222 \\ ${ }^{c}$ BioTools, Inc., 17546 Bee Line Hwy, Jupiter, Florida, 33458
}

\section{Supporting Information}

1. Atomic force microscope (AFM) imagining of lysozyme fibrils. The gelatinous phase of the incubated protein collected by centrifugation was re-suspended in $\mathrm{pH} 2.0 \mathrm{HCl}$ solution with a 1:400 dilution factor $(\mathrm{V}: \mathrm{V})$ and treated in ultrasonic bath for 15 minutes. The fibril suspension was then deposited onto freshly cleaved mica supports and incubated for 2 min followed by washing in de-ionized water and dried by nitrogen gas. The AFM imaging was performed on a NTEGRA spectra Raman spectroscopy SPM system (NT-MDT Co.,), in semi-contact mode. Super-sharp DLC silicon probes were used (nsg01-dlc: resonant frequency $150 \mathrm{kHz}$, stiffness $5 \mathrm{~N} / \mathrm{m}$ ). Initial amplitude of cantilever oscillations was in the range of $3 \sim 7 \mathrm{~nm}$. Set point was chosen at attractive regime.
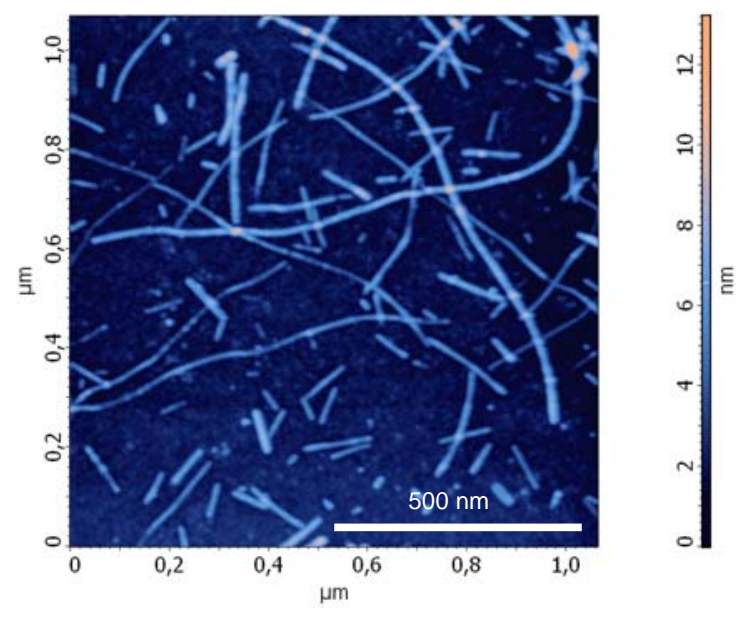

Figure S1. AFM topograph of lysozyme fibrils
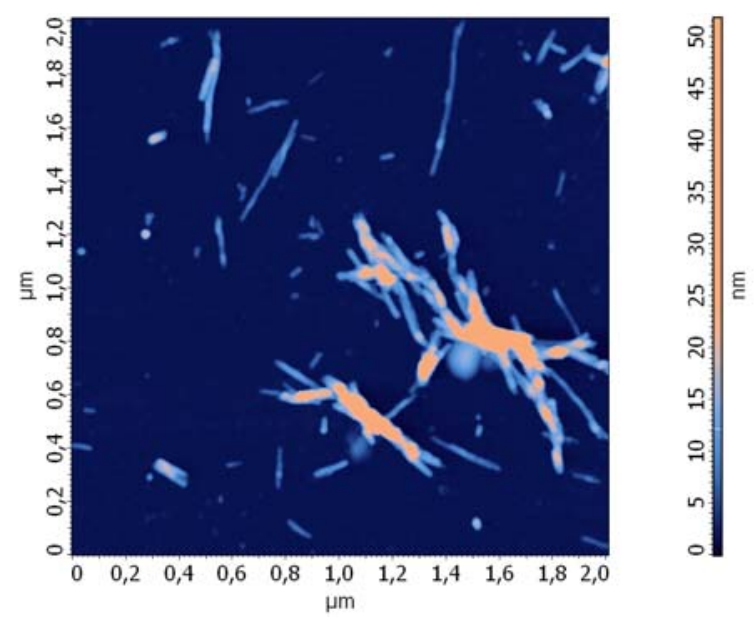

Figure S2. AFM topograph of insulin fibrils

2. Comparison of secondary structure composition of native bovine lysozyme and native insulin: Lysozyme has $\sim 62 \%$ random coil (PPII), $30 \% \alpha$-helix, and only $\sim 8 \% \beta$-sheet. Insulin has $42 \%$ random coil (PPII), $44 \% \alpha$-helix, and $14 \% \beta$-sheet. Both proteins have multiple S-S links. 

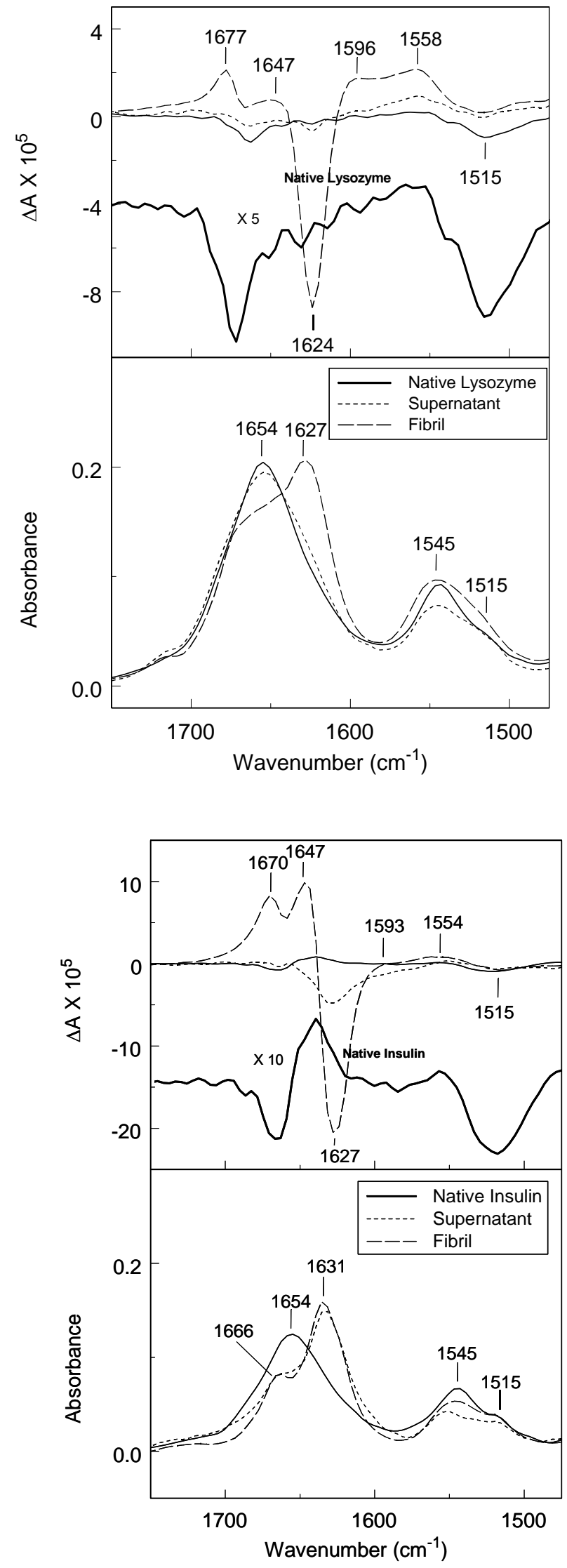

Figure S3. IR and VCD spectra of lysozyme fibril formation and growth at $\mathrm{pH} 2$, with heating at $65^{\circ} \mathrm{C}$ for two days, then separation by centrifugation into supernatant and gel phases, compared to native lysozyme at $\mathrm{pH} 2$, including expanded view of native lysozyme VCD. Spectra normalized to approximately equal integrated IR intensity.
Figure S4. IR and VCD spectra of insulin fibril formation and growth at $\mathrm{pH} 2$, with heating at $60^{\circ} \mathrm{C}$ for two hours, then separation by centrifugation into supernatant and gel phases, compared to native insulin at $\mathrm{pH} 2$, including expanded view of native insulin VCD. Spectra normalized to approximately equal integrated IR intensity. 
4. VCD and IR spectra of insulin fibers formed from a solution of concentration $10 \mathrm{mg} / \mathrm{mL}$ by heating at $60^{\circ} \mathrm{C}$ for four hours.

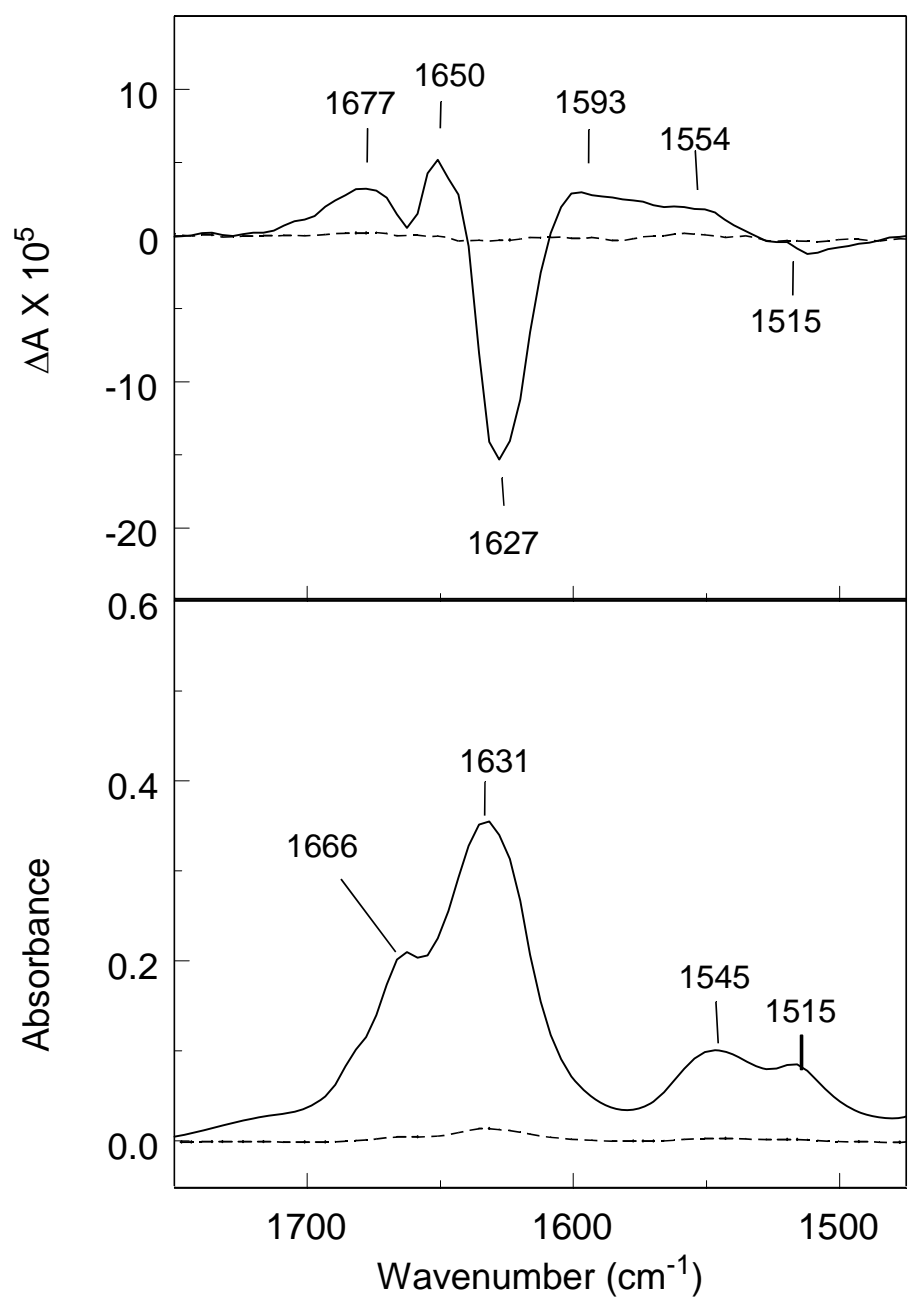

Figure S5. Insulin fibrils developed from native solution at a concentration of $10 \mathrm{mg} / \mathrm{mL}$, $\mathrm{pH} 2$, with heating for 4 hours at $60{ }^{\circ} \mathrm{C}$. Dashed line: The solution was heated for 4 hours and measured without centrifugation. Solid line: The solution was heated for 4 hours and centrifuged for 10 minutes at $10,000 \mathrm{rpm}$. The bottom gel phase was used for the IR and VCD measurements. All measurements were carried out at $8 \mathrm{~cm}^{-1}$ resolution for 1 hour. VCD and IR spectra have been solvent subtracted. The spectral features for both the IR and VCD are virtually the same as those presented in the published communication and hence the concentration of the initial solution used to grow the fibrils is not an important factor in the observed IR and enhanced VCD of the fibrils formed.

We feel that centrifugation over a 10 minute period to concentrate the dilute solution for VCD measurements did not compromise the results obtained for two reasons. First, we obtained the same IR spectra before and after centrifugation, and there is evidence for the same VCD emerging from the noise. Second, imaging fibrils with SEM or AFM is almost always preceded by a step of increasing the concentration so that a sufficient number of fibrils can be imaged. It is assumed that this concentrating step used prior microscopy does not alter the nature of the fibrils that were formed at low concentrations.

We checked the samples measured for orientation dependence of the sample in the cell that would occur if a liquid crystal phase developed in the gel. We find no linear or circular polarization dependence. The gel solutions appear to be isotropic, non-oriented solutions of fibrils or fibril assemblies that are randomly oriented. 


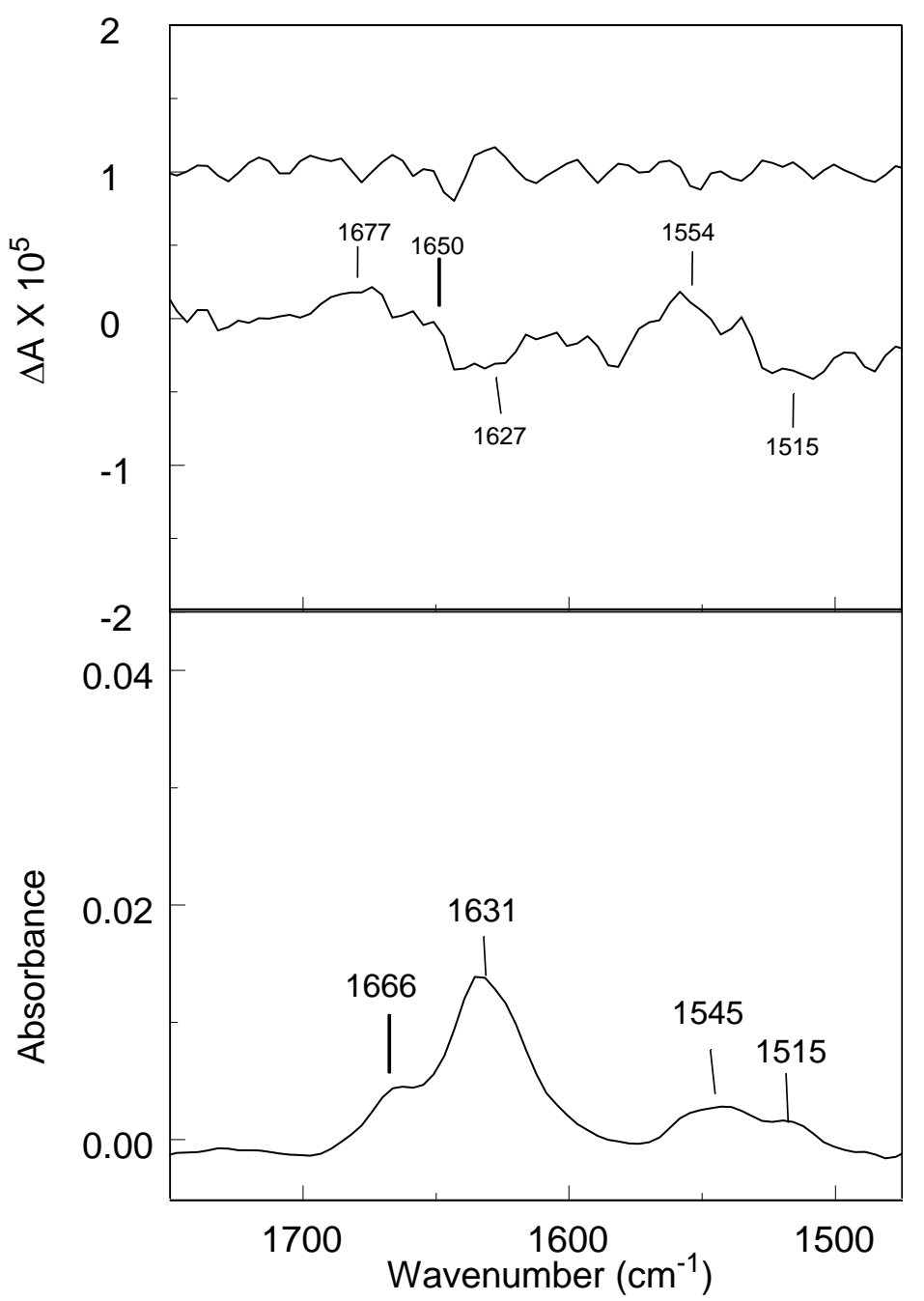

Figure S6. IR (lower), VCD (middle) and VCD noise (upper) spectra, presented on an expanded scale, of the insulin fibril solution measured without centrifugation shown as the dashed line IR and VCD spectra in Figure S5. The IR spectrum is nearly identical to the IR spectrum of the centrifuged gel in Figure S5, and the VCD spectrum is consistent with centrifuged gel VCD spectrum in Figure S5 although still somewhat obscured by noise. Note that no VCD spectrum of a protein solution in water has ever been measured with amide I absorbance peak with a value of only 0.02 , and yet here the VCD features are beginning to emerge.

Concerning the origin of large VCD intensities in protein fibril solutions, one might consider 1 ) protein conformation changes during heating, 2) the formation of the fibrils, and 3) the formation of a gel phase. A small conformational change, although it may aid in the formation of fibril, alone cannot be responsible for the order of magnitude increase in VCD intensities since hundreds of VCD spectra in various states of conformation show no indication of any dramatic increase in VCD intensities as reported here. As for gel formation, Figure 3 shows very large VCD intensities from a sample that was not centrifuged and has no separated or isolated gel phase. Hence the large increases in VCD intensity reported can safely be ascribed to the formation and development of protein fibrils. 